\title{
La focalisation « médiale » : pour un regard post- constructiviste sur les outils de communication
}

Medial Focalization: Toward a Post-Constructivist Perspective on

Communication Tools

\section{Franck Cochoy}

\section{OpenEdition \\ Journals}

Édition électronique

URL : http://journals.openedition.org/edc/5129

DOI : $10.4000 /$ edc. 5129

ISSN : 2101-0366

Éditeur

Université Lille-3

Édition imprimée

Date de publication : 1 juin 2013

Pagination : 77-92

ISBN : 978-2-917562-09-3

ISSN : $1270-6841$

Référence électronique

Franck Cochoy, «La focalisation «médiale » : pour un regard post-constructiviste sur les outils de communication », Études de communication [En ligne], 40 | 2013, mis en ligne le 01 juin 2013, consulté le 02 mai 2019. URL : http://journals.openedition.org/edc/5129; DOI : 10.4000/edc.5129

Ce document a été généré automatiquement le 2 mai 2019.

(c) Tous droits réservés 


\title{
La focalisation « médiale » : pour un regard post-constructiviste sur les outils de communication ${ }^{1}$
}

\author{
Medial Focalization: Toward a Post-Constructivist Perspective on
}

Communication Tools

Franck Cochoy

1 «On nous cache tout, on nous dit rien »; « on ne nous dit pas tout »; « les médias nous manipulent»: combien de fois avons-nous entendu ce genre de critique dans la vie courante mais aussi, sous des formes à peine plus sophistiquées, dans le monde académique? La mise en accusation ordinaire ou savante des médias, la dénonciation récurrente de la propension supposée des organes de presse à "travestir» cette « réalité » qu'ils sont censés « dévoiler » repose sur une double hypothèse : le monde serait divisé en deux parties, la réalité d'un côté, et sa construction sociale et culturelle de l'autre, et il existerait d'autres moyens d'accéder à la première sans l'aide de la seconde pour dénoncer éventuellement leur radicale dissemblance.

2 J'entends récuser l'une et l'autre de ces deux hypothèses au nom d'une conception différente des éléments impliqués. Grâce à plus de trente ans d'anthropologie des sciences et des techniques, nous savons désormais que la réalité n'est pas le point de départ, mais plutôt le résultat d'un long processus de traduction, d'inscription et de réinscription du monde, qui consiste à sélectionner certains éléments, à les articuler et à les montrer sous d'autres formes, de façon à ce que le monde puisse être transporté à distance, traduit et «re-présenté » (Latour, 1993). À cet égard, les médias ne sont qu'un médiateur parmi tant d'autres qui se donne pour mission ou qui a pour effet, comme eux, de produire des comptes rendus de la réalité. Comme Czarniawska (2011) l'a récemment montré à partir d'une enquête au cœur des agences de presse, les médias travaillent dur pour « agencer $»^{2}$ (plutôt que construire) les faits qui correspondent à leurs objectifs : ils trient, choisissent, classent, arrangent, soulignent et cachent les nombreux éléments qu'ils recueillent, déplacent, produisent et transforment. Mais cela ne veut pas dire pour 
autant ni que la réalité pourrait être connue « dans sa totalité » et " dans son intégrité ", c'est-à-dire qu'il existerait, à l'écart des médias, comptes rendus, archives, statistiques, récits ou autres travaux historiques, un référent indubitable et directement accessible ; ni à l'inverse que la réalité qui résulte de ces opérations n'est que " construite ", qu'il s'agit d'un simple artefact performatif, bref que les représentations médiatisées du réel sont réductibles à de purs effets de discours.

Les éléments discursifs et tangibles sont étroitement agencés : "les faits sont faits", comme le souligne Bruno Latour en reprenant un mot de Bachelard (Latour, 1991, 30) : les faits sont à la fois des données objectives (le fait comme substantif) et des choses fabriquées (fait comme participe). Au lieu de faire des médias une exception, de leur reprocher un type d'action dont l'on oublie qu'il est engagé dans toutes les autres formes de représentation du monde, y compris scientifiques, je suggère de considérer les médias à l'égal des sciences, et donc de faire de leur vice une vertu : je propose de prendre l'idée que les faits et les discours sont produits simultanément comme une raison de faire l'économie d'un débat sur la "véracité » de ce que rapportent les médias. Et je prends le risque de soutenir que cet argument est valide non seulement pour la presse générale qui supporte la circulation de l'information en démocratie, mais aussi pour l'une des catégories de médias a priori les moins défendables, la presse des affaires, c'est-à dire d'un outil de communication d'une organisation (l'entreprise ou l'organe de presse) à destination des organisations (les adhérents et/ou groupes professionnels associés) qui assume se donner pour mission moins d'informer le public que de soutenir délibérément certains acteurs, certains produits, certaines politiques et les intérêts associés.

Cette "façon de voir ", loin d'être générale, a des conséquences importantes, notamment en matière de méthodologie des sciences sociales. Pour qui veut décrire le monde, les médias s'offrent comme une source d'information particulièrement abondante, présente, commode, accessible. Il s'agit même parfois de la seule source disponible dans un grand nombre de domaines, notamment dans les cas où les archives ont disparu ou sont à l'inverse trop dispersées ou trop nombreuses pour être raisonnablement traitées par un seul chercheur. Dans des secteurs d'activité où les archives sont disséminées, difficiles d'accès, voire détruites, on trouve un très grand nombre de revues professionnelles qui ont patiemment opéré la chronique de leur domaine : revues pour l'ingénieur, bulletins d'entreprise, magazines représentant une industrie ou un produit particulier... C'est ce type de source, d'ailleurs largement utilisé en sciences sociales mais sans faire l'objet de justifications appropriées, dont je souhaite défendre ici la capacité à documenter efficacement l'évolution du monde dont elles parlent. J'entends montrer que l'on peut en faire un usage fiable, voire exclusif, sans occulter pour autant les intérêts et les effets de réel dont elles sont porteuses. Après avoir présenté, défini et justifié cette approche, que je propose de nommer « focalisation médiale », et précisé les modalités de son bon usage, au carrefour des notions de "vision centrale » et de "vision périphérique », je donne un exemple d'analyse historique appuyé sur la lecture d'un média dont je suis très éloigné dans le temps comme dans l'espace social: la revue américaine Progressive Grocer. Cette publication s'est donné pour mission, dans les années 1920, de promouvoir des techniques commerciales modernistes auprès des petits épiciers indépendants. 


\section{La focalisation médiale...}

5 Un lecteur inquiet mais bienveillant pourrait concéder que la presse des affaires constitue une source d'informations appréciable sur tel ou tel domaine d'activité, pourvu qu'on croise les propos qu'elle véhicule avec d'autres sources d'informations. Je voudrais soutenir au contraire, en exagérant un peu, moins par provocation que pour des raisons de clarté pédagogique, que cette précaution n'est même pas nécessaire : on peut faire de l'entrée par une source unique un parti pris volontaire, et la considérer comme un atout plutôt qu'une faiblesse en matière de connaissance des pratiques couvertes par les médias considérés.

6 Quels sont les avantages de l'entrée par le truchement d'un seul média, que ce mode d'accès exclusif soit contraint ou choisi? L'enjeu peut se concevoir dans les termes des modes de focalisation chers au critique littéraire fondateur de la narratologie, Gérard Genette (1972). Rappelons que ce dernier distinguait trois façons de narrer une histoire : la « focalisation interne », qui consiste à restreindre la narration au point de vue subjectif d'un personnage ; « la focalisation externe », qui consiste au contraire à envisager le récit du point de vue d'un observateur extérieur, telle une caméra qui suivrait le protagoniste ; et la "focalisation zéro", qui consiste à adopter un point de vue "omniscient ", surplombant, doté d'une connaissance totale tant des événements objectifs que des visions subjectives engagés dans le déroulement de l'histoire considérée.

7 On pourrait penser que la posture idéale, pour l'historien, réside nécessairement dans la recherche de la focalisation zéro, censée offrir une connaissance exhaustive, exacte et quasi divine de l'histoire. Pourtant, l'ironie veut que la quête du réalisme maximum se solde par un manque total de réalisme, puisqu'aucun des acteurs de l'histoire n'est doté d'une telle vision; or l'on comprend mal l'histoire si l'on néglige l'incertitude fondamentale qui anime ses acteurs, tant vis-à-vis de ce qui se trame autour d'eux que de ce qui les attend dans le futur. Faut-il alors, à l'inverse, rechercher la focalisation interne ou externe ? Ces dernières sont préférables, mais tandis que la première exige l'adoption du point de vue d'un seul témoin quand ici il s'agit plutôt de recueillir l'avis de milliers d'entre eux, la seconde nous prive de la subjectivité qui conditionne pourtant l'expérience réelle des acteurs.

8 Or l'usage des revues professionnelles me semble précisément permettre de surmonter ces deux difficultés en nous offrant le moyen d'expérimenter une nouvelle forme de focalisation, que je propose de nommer focalisation «médiale». Paradoxalement, le recours à cette terminologie vise moins à encombrer le lexique des sciences sociales par un nouveau jargon qu'à faire preuve de clarté! J'emprunte l'adjectif «médial » à la grammaire, où il désigne une lettre située au milieu d'un mot, pour lui donner un autre sens, proche de "médiatique », mais non substituable à ce dernier terme à mon sens trop connoté. Est médiale la focalisation propre à l'usage d'un média, ni plus, ni moins.

En passer par un journal nous donne accès à un point de vue certes particulier, mais non réductible à une seule instance comme c'était le cas avec la focalisation interne. En effet, le point de vue d'un média est un point d'observation partagé par beaucoup d'autres. La focalisation médiale consiste moins à changer de position qu'à changer de mode de vision : elle nous conduit à adopter non pas le regard d'un acteur particulier, mais plutôt l'effet que produit l'emploi d'une même "prothèse oculaire " accessible à un grand nombre d'acteurs. Tout se passe comme si l'on regardait quelque chose non pas « depuis 
les yeux » d'un personnage donné (cas de la focalisation interne), non pas d'en haut (cas de la focalisation zéro) ou de côté (cas de la focalisation externe), mais depuis un certain type de "lunettes " que lui-même mais aussi beaucoup d'autres sont susceptibles de chausser. Or en regardant le monde du point de vue d'un média particulier, on obtient une image très dissemblable de ce qu'offriraient les autres focalisations. Plus précisément, adopter le point de vue «médial » de la presse professionnelle nous permet de considérer le cours des affaires à travers le seul prisme véritablement disponible pour les acteurs, de se figurer ainsi ce que pouvaient observer, comprendre et ressentir les professionnels de loin les plus nombreux et les plus présents.

Bien sûr, le regard focalisé de façon médiale n'est pas suffisant en soi mais, paradoxalement et comme nous allons le voir, le complément nécessaire n'est pas non plus forcément à rechercher "à l'extérieur " : souvent, ce qui nous permet de ne pas nous laisser complètement absorber par la focalisation médiale nous est livré par le média luimême.

\section{... entre vision centrale et vision périphérique}

11 Le « défaut » de vision, au sens de manque et non d'imperfection, tient à la mobilisation d'un seul type de regard là où deux modes de cognition sont pourtant disponibles. Les spécialistes de la perception distinguent deux voies d'accès visuel à la réalité : la vision centrale qui centre l'œil sur un point particulier, objet de toute l'attention du sujet, s'oppose à la vision périphérique, qui fournit des impressions globales sur le reste de son champ de vision (Campbell \& Wright, 2012).

12 Pour pallier le défaut de vision, il convient donc au contraire d'appliquer une vision stéréoscopique, qui regarde à la fois « au centre » et « autour ». Les sciences sociales sont très sensibles aux vertus de la vision périphérique, notamment depuis l'essai de Bateson :

Centrés sur la poursuite du particulier, sur des buts étroits, nous ne sommes attentifs qu'à une fraction de la totalité, nous bloquons notre vision périphérique, et nous agissons sans regarder l'image d'ensemble (Bateson, 1994 : 134, cité dans Campbell and Wright, 2012, ma traduction).

13 Toutefois, il importe de souligner que l'enjeu de la distinction entre les deux formes de perception ne consiste nullement à abandonner la vision centrale pour la vision périphérique. En effet, quitter un point focal pour un élément hors champ, c'est simplement changer d'objet d'attention, "regarder ailleurs", et donc revenir à cette vision centrale dont l'on croyait s'écarter, avec pour seule conséquence le risque de "perdre le fil », de se fourvoyer dans les méandres de la «distraction». Les visions périphérique et centrale n'ont de réel intérêt qu'à la condition d'être combinées, comme le souligne Stark :

[La vision périphérique] est vitale pour les organisations. Si l'horizon stratégique est réduit, au sens où le futur est proche mais très imprévisible, alors vous ne devez pas être bloqué en regardant simplement devant vous mais vous devez aussi vous montrer attentif au mouvement qui se produit autour de vous. La vision périphérique permet de prendre conscience d'un tel mouvement (Stark dans Harrington, 2010, ma traduction).

La promotion de cette vision duale pose tout de même un problème : l'opération conduitelle, plus ou moins implicitement, à postuler une différence de capacités perceptives entre chercheurs et acteurs? Oui et non. Non, au sens où il n'y a pas de différence en soi : 
le chercheur n'est évidemment pas fait d'une autre chair que celle de ses informateurs ; le premier ne dispose évidemment d'aucun privilège visuel et cognitif dont les seconds seraient privés. Mais oui, il existe souvent une différence entre chercheurs et acteurs, si l'on se réfère aux conditions d'exercice professionnel des uns et des autres : tandis que les acteurs sont la plupart du temps immergés dans l'action, pris par le cours de leurs urgences, absorbés par le règlement de problèmes prioritaires, les chercheurs sont extérieurs à l'action considérée; partant, ils ont davantage de temps, et donc la possibilité de prendre du recul, de revoir d'une autre façon les même matériaux, de revivre les mêmes actions. Ils peuvent, en particulier, décaler doublement leur regard dans le temps et dans l'espace, faire défiler l'action au ralenti d'une part, et regarder davantage " en marge » des scènes principales d'autre part. Les chercheurs se trouvent ainsi en mesure d'identifier ce qui échappait le plus souvent aux acteurs ordinaires; ils peuvent doubler la vision centrale des acteurs avec la vision périphérique permise par les opérations de recherche.

La focalisation médiale consiste ainsi à adopter le point de vue d'un média pour suivre l'action du point de vue du regard que ce média offre à un ensemble d'acteurs sur la réalité, tout en croisant les visions centrale et périphérique offertes par le même matériau.

\section{Des effets et du bon usage de la focalisation médiale : l'exemple de Progressive Grocer}

16 Maintenant que nous savons en quoi consiste la focalisation médiale et quelles sont les conditions d'exercice susceptibles de lui donner sens, je voudrais donner un exemple de mise au travail de cette notion dans les conditions définies. L'exemple que je retiens est celui de Progressive Grocer, un magazine professionnel mensuel fondé en 1922 et censé représenter les évolutions du commerce à destination des épiciers indépendants, à l'époque où ces derniers sont menacés par le développement des chaînes de magasins (qui disposeront en 1927 de leur propre revue, Chain Store Age) et bientôt par les supermarchés (qui auront également leur propre organe de presse, Supermarket Merchandising, lancé en 1936). Progressive Grocer fonctionne à ses débuts sur la logique de la presse gratuite : il s'agit non pas de l'émanation d'une corporation mais d'une entreprise de presse indépendante, dont les publications sont offertes aux épiciers et financées par la publicité d'entreprises qui vendent des fournitures pour magasins (caisses enregistreuses, étagères, etc.), de la presse magazine (Companion, Ladies Home Journal, Life...) qui y communique dans l'espoir de vendre aux épiciers son espace publicitaire, et de fabricants de produits alimentaires, qui essaient de convaincre les épiciers de mettre leurs produits à disposition de leur clientèle. Progressive Grocer est ainsi « une affaire en soi », qui essaye d'imposer sa propre médiation entre les fabricants et les détaillants ${ }^{3}$.

Il est raisonnable de postuler l'existence d'un décalage structural entre ce que montre cet organe de presse (qui en tant que "vitrine des vitrines ", fait profession de montrer les plus innovantes, et mérite donc bien son nom de «Progressive Grocer »), et l'état des autres magasins, dont on peut supposer qu'un grand nombre étaient beaucoup moins « avancés » que ceux que la revue met généralement en avant. En effet, si cette dernière nous donne une chance de saisir de façon relativement précise la chronologie des 
innovations en relayant les publicités chargées de les lancer, elle est beaucoup moins fiable pour se faire une idée du moment, du degré et des modalités de leur adoption.

Mais pour postuler l'existence d'un tel décalage, avons-nous besoin de regarder ailleurs que dans le média lui-même? On le pourrait, non pas en accédant à la "réalité des choses ", mais en passant par d'autres formes de médiation, comme des statistiques gouvernementales par exemple, ou des travaux académiques. Je l'ai d'ailleurs fait et c'est évidemment très instructif ${ }^{4}$. Mais ce n'est pas non plus nécessaire, dans la mesure où le même type de renseignement peut se repérer à la périphérie d'une lecture «centrale » de Progressive Grocer. L'un des aspects originaux de la revue est en effet d'articuler différents types de représentations (des statistiques sur l'état du commerce, des articles militants, des publicités, des témoignages, des reportages, des photographies...) dans lesquels on peut directement repérer différentes «torsions » du référent qu'elle se charge de mettre en scène à titre principal. Ainsi, la traque des « décalages » dont l'on accuse la presse, loin d'exiger le détour par une "réalité » utopique inaccessible, ou même loin de forcément requérir l'usage de sources alternatives ou secondaires, peut au contraire se repérer directement dans la représentation « médiale » de l'outil de communication considéré.

Par exemple, la prise en compte de quelques statistiques austères perdues au milieu des articles modernistes et promotionnels qui militent à longueur de pages, à grand renfort d'images spectaculaires et d'arguments enthousiastes en faveur de nouvelles formes d'organisation des ventes, montre que la forme classique du service au comptoir fut plutôt longue à céder sa place aux arrangements modernes du semi libre-service et du libre-service. En 1939, la vente au comptoir a depuis longtemps disparu des pages de Progressive Grocer (à l'exception de cas très rares dévoués au rappel du «bon vieux temps »). Pourtant, un graphique publié en 1949 au détour d'un article sur l'intérêt futur des supérettes en libre-service montre que le service au comptoir était encore la solution utilisée par presque la moitié des magasins, avec seulement $13 \%$ d'entre eux utilisant le libre-service (les $42 \%$ restants relevant de la solution hybride du semi libre-service); c'est seulement en 1947 que le pur libre-service devint la solution majoritaire (1949, 04, $59)^{5}$.

20 On pourrait penser que la logique d'offre inhérente au gratuit est responsable d'un tel décalage entre les représentations disponibles puisque l'envoi d'une publication non sollicitée ne présume en rien de sa lecture; partant, nombre d'épiciers seraient restés insensibles aux innovations modernistes promues par Progressive Grocer. Pourtant, la revue revendique dès 1930 une diffusion auprès de 68000 détaillants $(1930,08,2-3)$ soit près du tiers des 250000 épiciers indépendants de l'époque (1930, 02, 14-15), un lectorat considérable qu'aucune autre forme de diffusion n'aurait sans doute eu la chance d'atteindre. Même s'il est vraisemblable qu'un fort pourcentage des destinataires ont ignoré la revue ou ne l'ont regardée que très distraitement, comme pour toute publication publicitaire (Canu, 2009), l'hypothèse d'un impact réel important auprès du monde des épiciers semble la plus probable, comme en attestent d'ailleurs la croissance de la revue, la confiance continue que lui ont témoigné les annonceurs et la migration progressive des innovations présentées dans les espaces de vente photographiés.

21 La raison du décalage entre ce que la revue montre massivement et d'autres traductions de l'état du commerce plus discrètes mais malgré tout relayées dans ses pages doit donc être cherchée ailleurs, du côté de son style et de sa mission. Afin de moderniser les épiceries, Progressive Grocer utilise une rhétorique particulière: l'idée est de faire impression (sur les pages et sur le public qui les lit) ; il s'agit d'encourager les avancées des 
nouvelles techniques de merchandising en montrant le présent comme image de temps révolus (cf. la vente au comptoir dans les années 1930), en présentant certaines des rares initiatives préfigurant le futur souhaité comme l'état présent des affaires ou en combinant ces deux stratégies sous la forme de la mise en scène de quelques évolutions spectaculaires via l'usage de la figure dramatique de l'«avant/après ", comme dans l'image suivante :
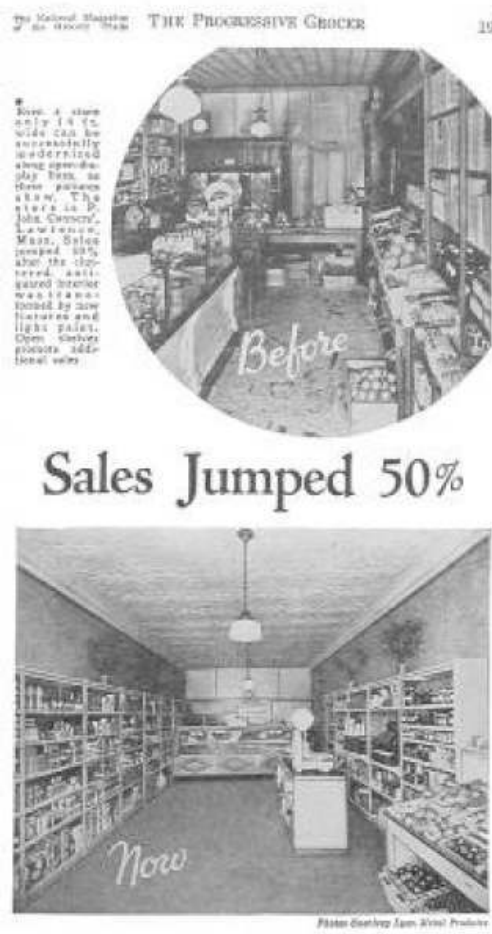

Figure 1 : D'hier à aujourd'hui, les ventes ont bondi de $50 \%(1936,01,19)$

Cependant et paradoxalement, le spectacle d'un tel décalage ne disqualifie pas la capacité de la revue à représenter le monde dont elle parle. Au contraire, il souligne son aptitude à «re-présenter» efficacement ce monde, au sens latourien du verbe (1993): la revue présente le monde une deuxième fois, et différemment, de telle sorte que les acteurs puissent reconnaître le monde qu'on leur montre comme étant le leur, et ainsi ajuster leurs pratiques en conséquence.

La re-présentation de l'économie épicière qu'opère la revue est d'autant plus efficace qu'elle s'adresse à un épicier dont la vue est aussi limitée que celle du public de Lippmann (2008). En effet cet épicier, isolé et sédentaire dans son magasin, sortant peu et travaillant beaucoup, ne voit ses concurrents que de loin, ou depuis sa vitrine, tandis que cette brochure qu'il reçoit chaque mois lui offre, par contraste, une vue omnisciente ${ }^{6}$ sur le monde de l'épicerie en général; une vue qu'il ne peut que recevoir comme une image adéquate du monde extérieur. Progressive Grocer lui présente des milliers d'expériences, d'équipements et de discours qui constituent pour lui autant de pratiques et d'identifications possibles, voire nécessaires. Progressive Grocer conduit ainsi notre épicier à prendre l'avant-garde de la distribution comme l'état présent du commerce, et donc par conséquent son propre état pour l'arrière-garde. Puisque le " pauvre homme » s'inquiète de la concurrence et des innovations présentées dans la revue, il n'a pas d'autre choix, s'il veut rester dans la course et rester digne de sa profession, que d'engager sa personne, son 
magasin et ses clients dans ce mouvement de modernisation «irrésistible » qui apparemment a déjà emporté la plupart de ses confrères. L'épicier est incité à acheter et à mettre en place les meubles, les produits et les techniques qui lui sont présentés comme le moyen de vaincre ses inquiétudes, éviter la faillite et accomplir ses projets.

Cette tension entre ce que la revue montre le plus souvent et d'autres états plus systématiques du commerce qu'elle relate à l'occasion peut-être retracée à partir d'autres décalages significatifs que l'on observe entre les articles et les nombreuses publicités et photos qui y sont reproduits.

Les publicités publiées dans Progressive Grocer sont souvent en avance sur les articles qui aborderont plus tard les équipements qu'elles présentent. De nouvelles pratiques et de nouveaux équipements émergent ainsi parfois sous la forme d'" externalités picturales ", c'est-à-dire d'éléments présents dans le champ des éléments photographiés, mais étrangers à l'objet même de la photographie. C'est notamment le cas de l'usage des chariots de supermarché, comme le montrent de façon particulièrement spectaculaire les deux clichés suivants.
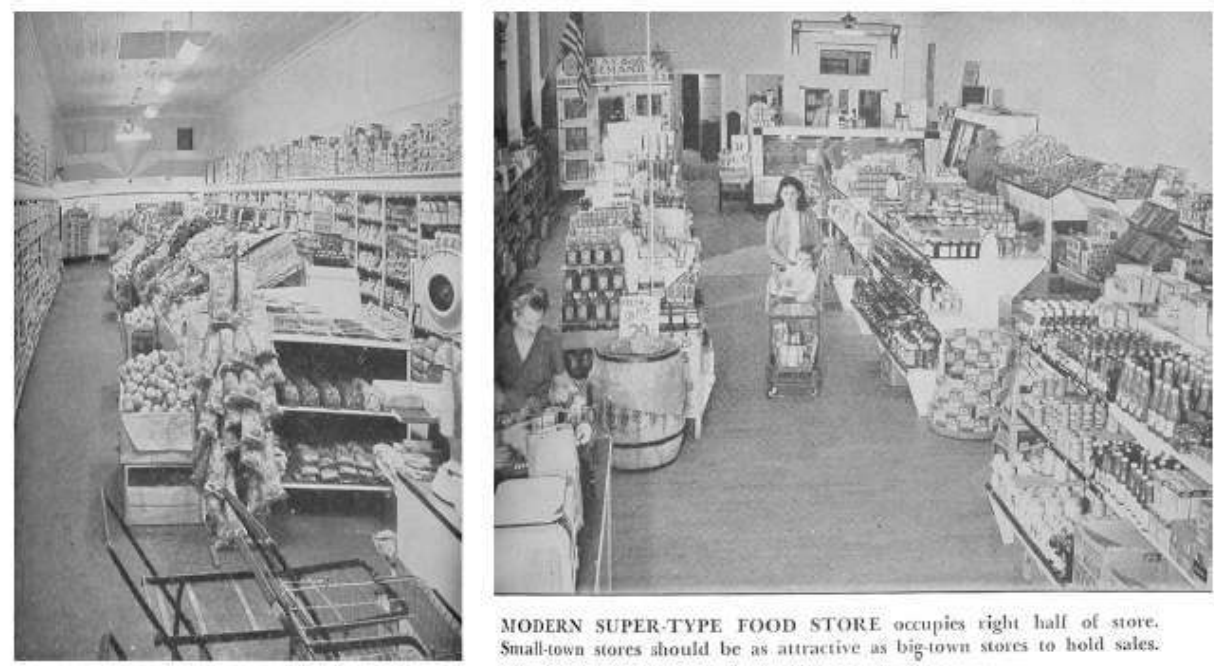

MODERN SUPER-TYPE FOOD STORE occupies right half of store.

Figure 2 : À gauche: "Remodeler des magasins étroits » $(1944,09,84-85)$;

à droite: "Le magasin d'alimentation moderne de type super » $(1945,10,103)$

La photographie de gauche vient illustrer un article intitulé «Comment remodeler des magasins étroits? Les idées sont ici ». Cet article est destiné comme son nom l'indique à donner des astuces d'aménagement pour tirer le meilleur parti de l'espace exigu des épiceries de l'époque. Le texte s'intéresse à tout (rayons, comptoir, stockage...), sauf aux chariots de supermarché, et ne mentionne évidemment pas ceux d'entre eux qui encombrent le premier plan de son illustration, mais qui se situent à la périphérie des éléments qu'il désigne, tous situés à l'arrière-plan (" rayons à la profondeur étendue ", " murs gris clairs », « habillage intérieur bleu », " meubles blancs »). Or, ces chariots sont vendus depuis 1936 avec comme principal argument commercial qu'ils sont pliables, un avantage censé favoriser leur adoption dans un marché de l'épicerie justement dominé à l'époque par ces boutiques minuscules où l'espace est compté. La pratique montre qu'en dépit des arguments publicitaires les chariots pliables ne sont jamais pliés, et vont même jusqu'à manger l'espace d'un magasin que l'on présente justement comme modèle des règles à suivre en matière d'optimisation de la faible surface de vente ! (Cochoy, 2009). 
De même, la photographie de droite met l'accent sur l'évolution vers des magasins de type "supermarché ", et situe très explicitement dans sa légende le changement à suivre dans « la partie droite du magasin ", là où l'on voit des allées, des gondoles, des étagères basses facilement accessibles en libre-service. L'article oublie en revanche complètement non seulement le chariot qui apparait au centre, mais surtout l'usage du dispositif, dont le panier supérieur est occupé par un bambin. Dans l'image, donc, le centre de l'attention est à la périphérie et l'élément périphérique oublié se trouve au centre. Cet " aveuglement », loin d'être restreint à cette image, est endémique dans Progressive Grocer : depuis leur introduction en 1936, les chariots envahissent à longueur de pages les annonces et les photographies que publie la revue, mais cette dernière, obsédée par l'aménagement fixe des magasins, les plans, les meubles, le "display ", ne les remarque pas, sauf, significativement, lorsqu'elle peut les présenter à l'occasion comme étagères d'appoint. Les consommateurs détournent les chariots pour s'en servir de poussettes, au risque non seulement d'exposer leur progéniture à des accidents, mais surtout (du point de vue de l'épicier!) de faire perdre la moitié de sa capacité de transport et de compromettre ainsi l'ensemble du dispositif du libre-service. Il faudra attendre 1947 pour que les fabricants s'emparent du problème et tentent de lui trouver des solutions (Cochoy, 2008). On le voit, il est bien difficile de prêter attention à ces petites choses qui prolifèrent dans notre champ de vision quand notre attention a pris l'habitude de regarder ailleurs, quand bien même ces "détails » auraient pourtant la capacité de changer toute une économie. Alors que Progressive Grocer, jusqu'à la fin de la seconde guerre mondiale, passe en revue l'ensemble des modalités du libre-service à l'exception $\mathrm{du}$ chariot, c'est très certainement ce dernier plus que tout autre dispositif qui a soutenu l'avènement de la distribution de masse, en permettant l'essor du libre-service, des achats accrus, des courses moins pénibles, plus longues, plus massives et plus espacées, et donc la massification des ventes, l'extension des surfaces commerciales et la concentration du secteur de la distribution. Le cas des chariots montre non seulement que les effets de torsion du référent sont repérables au cœur même de la focalisation médiale, sans nécessiter de détour extérieur, mais aussi que l'illusion peut être involontaire, et même que l'illusionniste peut être parfois victime de sa propre expertise.

\section{Conclusion}

Adopter la focalisation médiale, c'est-à-dire chausser les lunettes communicationnelles que certaines organisations proposent à leurs lecteurs pour " voir » et " colorer » mieux et autrement le monde qui les intéresse, permet donc d'éprouver avec les acteurs ce regard, de "voir ce que cela fait de voir les choses» via le média considéré, donc de revivre par empathie ce regard et son effet, qui ont fait l'histoire, agencé les choses et les gens, fait bouger le monde.

En défendant l'entrée dans l'histoire des affaires par la focalisation médiale j'ai tenté d'être cohérent, en instruisant la critique mais aussi la pragmatique du média à partir du média lui-même. J'ai montré que ce dernier produit en même temps son propre discours et les «torsions » qui permettent de le mettre à distance. Il ne faut donc pas rejeter mais au contraire embrasser les effets de déformation propres aux médias qui, en déformant leur référent, donnent paradoxalement un accès fidèle à la réalité même des modes de représentation du social. Il ne faut pas avoir peur d'entrer dans le monde par ces médias qui le constituent, tout en prenant grand soin de se doter de la vision périphérique qui 
permet, au passage, de mesurer la force, l'orientation et les dangers de ce courant constitutif.

\section{BIBLIOGRAPHIE}

Bateson M. C., (1994), Peripheral visions : learning along the way, New York, HarperCollins Publishers, $256 \mathrm{p}$.

Çalışkan K. et Callon M., (2010), Economization, part $2:$ a research program for the study of markets, in Economy and Society, vol. 39, $\mathrm{n}^{\circ} 1$, pp. 1-32.

Campbell D. et Wright J., (2012), The importance of peripheral vision in evaluative practice, en ligne : http://ucanr.org/sites/UC_CCP/files/125957.pdf, consulté le 18 janvier 2013.

Canu R., (2009), La manipulation des documents publicitaires. Contribution à une sociologie du travail marchand, in Revue Française de Socio-Économie, n 3, pp. 147-167.

Cochoy F., (2008), Hansel and Gretel at the grocery store: Progressive Grocer and the little American consumers (1929-1959), in Journal of Cultural Economy, vol. 1, n² 2, pp. 145-163.

Cochoy F., (2009), Driving a shopping cart from STS to business, and the other way round. On the introduction of shopping carts in American grocery stores (1936-1959), in Organization, vol. 16, $\mathrm{n}^{\circ} 1$, pp. 31-55.

Czarniawska B., (2011), Cyberfactories, How news agencies produce news, Cheltenham, Edward Elgar, $240 \mathrm{p}$.

Genette G., (1972), Figures III, Paris, Seuil, 286 p.

Harrington B., (2010), The Sense of dissonance - An interview with David Stark, 14 avril 2010, en ligne : http://thesocietypages.org/economicsociology/2010/04/14/the-sense-of-dissonance-aninterview-with-david-stark/, consulté le 18 janvier 2013.

Latour B., (1991), Nous n'avons jamais été modernes. Essai d'anthropologie symétrique, Paris, La Découverte, 205 p.

Latour B., (1993), Le topofil de Boa-Vista, in Conein B., Dodier N. et Thévenot L. (dirs.), Les objets dans l'action, De la maison au laboratoire, Raisons Pratiques, $\mathrm{n}^{\circ} 4$, Paris, éditions de l'E.H.E.S.S. pp. 187-216.

Latour B., (2007), La connaissance est-elle un mode d'existence ? Rencontre au Muséum de James, Fleck et Whitehead avec des fossiles de chevaux, in Debaise D. (dir.), Vie et expérimentation : Peirce, James, Dewey, Paris, Vrin, pp. 17-44.

Leymonerie C., (2011), L'aluminium, matériau des arts décoratifs à l'Exposition Internationale de Paris en 1937, in Cahiers d'histoire de l'aluminium, nº 46-47, pp. 9-49.

Lippmann W., (2008), Le public fantôme, Paris, Démopolis, [1925], 188 p.

Stark D., (2009), The Sense of dissonance. Accounts of worth in economic life, Princeton and Oxford, Princeton University Press, 245 p. 


\section{NOTES}

1. Je remercie chaleureusement le Centre for Retailing de l'Université de Göteborg pour son soutien, Progressive Grocer pour m'avoir accordé la permission de reproduire les images qui illustrent cette publication, Roland Canu ainsi que les lecteurs anonymes de la revue pour leurs remarques et suggestions.

2. Cf. Çalışkan and Callon, 2010.

3. Dans le cadre de mes travaux sur les médiations marchandes, et à l'occasion de deux missions de recherche à Berkeley (2006) et Chicago (2013), j'ai dépouillé l'ensemble des numéros de la revue sur la période 1922-1959, en retenant une approche "d'archéologie contemporaine " attentive autant aux textes qu'aux objets du passé, notamment tels qu'ils apparaissent dans l'iconographie (Cochoy, 2009).

4. D'une façon générale, comparer comment deux médiations distinctes rendent compte d'un même objet est passionnant, comme l'a relevé Latour (2007) à propos des fossiles de chevaux.

5. Dans la suite du texte, les renvois entre parenthèses indiquent pour chaque document cité l'année, le mois puis les pages de la revue Progressive Grocer.

6. Les médias offrent une focalisation zéro par procuration, mais le leurre consiste évidemment à confondre focalisation médiale (pragmatique) et focalisation zéro (utopique).

\section{RÉSUMÉS}

L'article soutient qu'il est possible d'aborder la description du monde à partir de ce que nous en montrent les médias, dans la mesure où le monde, loin d'être réductible à la prétendue "réalité extérieure » ou au contraire à une pure « construction sociale », est plutôt le produit performatif des médiations qui sont censées le véhiculer. Il s'agit donc de défendre l'adoption d'une «focalisation médiale », complémentaire vis-à-vis des focalisations «interne », « externe » et «zéro» de G. Genette, qui consiste à adopter le point de vue partagé ou partageable que certaines organisations proposent à leurs lecteurs pour « voir » et « colorer » mieux et autrement les objets qui les intéressent. L'exemple choisi est celui de Progressive Grocer, un magazine américain destiné aux épiciers de l'entre-deux-guerres. En chaussant les « lunettes » offertes par ce magazine, l'article propose d'éprouver avec les acteurs le regard qu'ils ont porté sur leur univers professionnel, et donc de revivre par empathie ce regard, son effet, ses promesses mais aussi ses dangers.

This paper argues that it is possible to approach the description of the world from what media show of it to the extent that far from being reducible to external reality or on the contrary to a pure social construction, the world consists of the performative effect of the mediations that are supposed to convey it. The paper makes a claim for the adoption of a medial focalization that would complement Gérard Genette's internal, external, and zero focalizations. This approach proposes to adopt the shared (or sharable) view that some organizations invite their public to see and color the items in which they are interested. The example chosen is that of the Progressive Grocer, a trade magazine aimed at American grocers between the two world wars. Looking through the lens provided by this magazine, the paper proposes to test along with the actors the 
view they took of their professional universe and thus to revive this view, its effect, and its promises but also its risks.

INDEX

Keywords : medial focalization, representation, constructivism, press, market, history

Mots-clés : focalisation médiale, représentation, constructivisme, presse, marché, histoire

\section{AUTEUR}

FRANCK COCHOY

CERTOP - UMR CNRS 5044, Université Toulouse 2

Franck Cochoy, Université Toulouse 2, CERTOP (UMR CNRS 5044). Adresse électronique : cochoy@univ-tlse2.fr. 